\title{
First-Principles Materials Study for Spintronics: MnAs and MnN
}

\author{
R. de Paiva ${ }^{1}$, J. L. A. Alves ${ }^{2}$, R. A. Nogueira ${ }^{1}$, J. R. Leite ${ }^{3}$, and L. M. R. Scolfaro ${ }^{3}$ \\ ${ }^{1}$ DF-UFMG, CP 702, 30.161-970 Belo Horizonte, MG, Brazil \\ ${ }^{2}$ DCNAT-UFSJ, CP 110, 36.300-000 São João del Rei, MG, Brazil \\ ${ }^{3}$ DFMM-IFUSP, CP 66318, 05389-970 São Paulo, SP,Brazil
}

Received on 31 March, 2003

\begin{abstract}
We report ab-initio all electrons density- functional calculations for the electronic structure of the compounds MnAs and $\mathrm{MnN}$, in the zinc-blende phase. They are potential materials for use in fabrication of new functional semiconductors taking advantage of the spin degree of freedom. The aim is the establishing of the semiconductor spin electronics (spintronics) as a practical technology [H. Ohno, Semiconductor Science and Technology 17, 4 (2002).]. We compare results obtained using the theoretical approaches LDA (Local Density Approximation) and GGA (Generalized Gradient Approximation). The calculations are spin-polarized and we follow the evolution of the band structures as a function of lattice parameter. We compare also the evolution of the density of states of the majority-spins and of the minority-spins. We conclude that, depending on the lattice parameter, both materials may be half-metallic, therefore showing conduction by charge carriers of one spin direction exclusively: the majority- spin band is continuous, while the minority-spin has a gap. Both materials reach a total magnetization of the order of $4 \mu_{B}$. MnN changes from paramagnetic to ferromagnetic with the increase of the lattice parameter.
\end{abstract}

\section{Introduction}

The Mn atom as a constituent of the so called diluted magnetic semiconductors (DMS) is nowadays the object of many experimental and theoretical investigations [1-12]. In the present work we compare the electronic and magnetic properties of MnAs and $\mathrm{MnN}$ in the hypothetical zincblende $(\mathrm{ZB})$ phase. Although the $\mathrm{ZB}$ is not the ground-state structure for these materials [13-19], we find interesting to study them in the $\mathrm{ZB}$ structure, since in it the $\mathrm{Mn}$ atoms find the same local environment as in diluted alloys, such as $\mathrm{Ga}_{1-x} \mathrm{Mn}_{x}$ As or $\mathrm{Ga}_{1-x} \mathrm{Mn}_{x} \mathrm{~N}(\mathrm{x}<0.07)$. The tetrahedral coordination of the $\mathrm{Mn}$ atoms seems to play an important role in the understanding of the properties of the III-V DMS. Therefore, we study the compounds $\mathrm{MnAs}$ and $\mathrm{MnN}$ in the $\mathrm{ZB}$ structure in order to have a better understanding of the nature of the chemical bond of Mn tetrahedrally coordinated with As or $\mathrm{N}$ in the III-V DMS.

\section{Theoretical framework}

We carry out first-principles band structure and total-energy calculations within the framework of the density functional theory [20] and compare the results for two approaches, namely the local density approximation (LDA) and the generalized gradient approximation (GGA). We use the fullpotential linearized-augmented plane-wave method as implemented in the Wien2k code [21] and applied to a 2-atoms unit cell. The muffin-tin radii adopted were 2.0 a. u. (Mn), 1.89 a. u. (As) for MnAs and 1.75 a. u. (Mn), 1.44 a.u. (N) for MnN. The Rkmax parameter was taken as 8.0 and the angular momentum $1=10$ for the expansions inside the spheres. The total energy and eigenvalues were converged to $10^{-5} \mathrm{eV}$, and $10^{-4} \mathrm{eV}$, respectively. The calculations were performed with spin-polarized potentials in order to analyze the ferromagnetic phases of the compounds. We follow the behavior of the band structures, density of states, fermi level, and magnetic moments as a function of the lattice parameter.

\section{Results and discussion}

In Fig. 1 we show the behavior of the cell magnetic moment as a function of lattice parameter. One can see that the magnetic moments for $\mathrm{MnN}$ are zero at the equilibrium lattice parameters $\left(a_{o}=4.19 \AA(\mathrm{LDA})\right.$, and $\left.4.30 \AA(\mathrm{GGA})\right)$; for MnAs the magnetic moments are $2.5 \mu_{B}$ and $4.0 \mu_{B}$ respectively at the equilibrium lattice parameters $a_{o}=5.32 \AA(\mathrm{LDA})$ and $5.71 \AA$ (GGA). It is noticeable that the magnetization undergoes large changes induced by small changes in the lattice parameters. The LDA values always tend to be lower than the GGA ones. In both approaches it is predicted that expansions (compressions) tend to turn the materials more ferromagnetic (paramagnetic). In the case of $\mathrm{MnN}$ the ferromagnetic phase should appear at moderate expansions over the equilibrium lattice parameter. This behavior should be understandable by looking at the band structures and density of states. We note that the magnetic coupling between the $\mathrm{Mn}$ atom and the As atom or $\mathrm{N}$ atom is always antiferromagnetic. It can be seen in Fig. 1 that the magnetic moments increase as the lattice parameters increase and tend to saturate at the value $4.0 \mu_{B}$ in both cases. The saturation of the magnetization at the value $\sim 4.0 \mu_{B}$ 


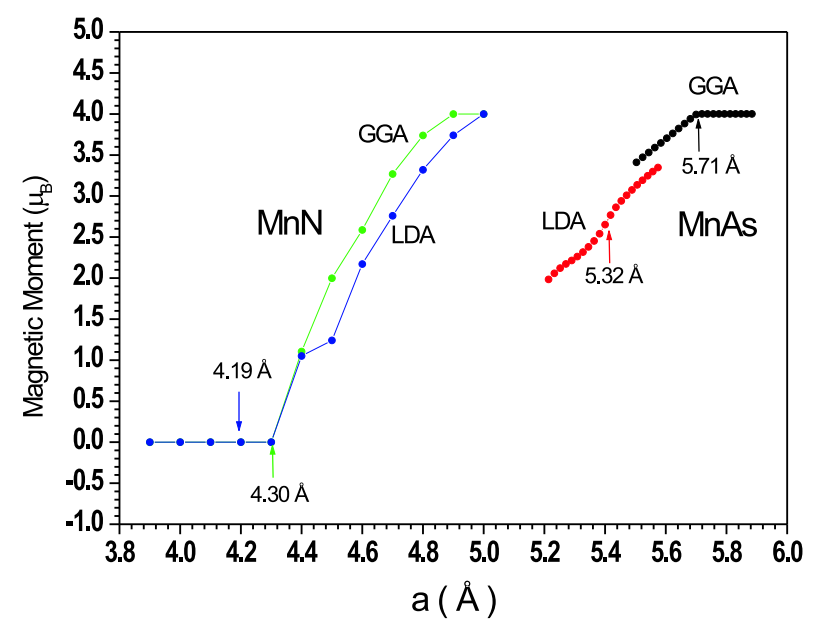

Figure 1. Magnetization, per unit cell, for the compounds $\mathrm{MnN}$ and MnAs as a function of the lattice parameter in both approaches LDA and GGA. The arrows indicate the equilibrium lattice parameters.

may be understood on the basis of a model of charge transfer from Mn to the neighbors' $4 p(A s)$ and $2 p(N)$ shells [10]. This charge transfer is not complete due to the strong interaction originating from the $\mathrm{p}-\mathrm{d}$ hybridization. As the unit cell is expanded this hybridization is reduced and the $\mathrm{Mn}$ atom tend to a configuration with four valence electrons which, following Hund's rule, reaches a magnetic moment of $4.0 \mu_{B}$. On the other hand this p-d interaction leads to quite large negative polarization of the anions $\left(\sim-0.10 \mu_{B}\right)$, being larger for As than for $\mathrm{N}$. As the lattice parameters increase the spin polarization turn to be effective and this is reflected in the electronic band structures and density of states. In Fig. 2 we show the band structures and density of states for $\mathrm{MnN}$ and MnAs, both at the equilibrium lattice parameter and calculated in the GGA approach. The band structures exhibit the levels originated from atomic states $\Gamma_{1}(\mathrm{~N}-$ $2 \mathrm{~s}$, As-4s), $\Gamma_{15 p}(\mathrm{~N}-2 \mathrm{p}, \mathrm{As}-4 \mathrm{p}), \Gamma_{12}(\mathrm{Mn}-3 \mathrm{~d})$ and $\Gamma_{15 d}(\mathrm{Mn}-$ $3 \mathrm{~d}$ ), at the $\Gamma$ point. In the case of $\mathrm{MnN}$, the $\mathrm{N}-2 \mathrm{p}$ valence band appears as the first $\Gamma_{15 p}$ level, below the Fermi level, $\mathrm{E}_{F}$, for both minority-spin and majority-spin systems. The $\Gamma_{12}$ bands originated from $\mathrm{N}-3 \mathrm{~d}$ states are entirely occupied, while the $\Gamma_{15 d}$ bands are empty. On the other hand, in the case of MnAs the minority-spin and majority-spin systems have different band structures. For the minority-spin system the low lying $\Gamma_{15 p}$ band is As- $4 p$ in origin, and the $\Gamma_{12}$ and $\Gamma_{15 d}$ bands are empty. For the majority-spin system the low lying $\Gamma_{15 d}$ band is Mn- $3 \mathrm{~d}$ in origin, the $\Gamma_{12}$ band is occupied and the $\Gamma_{15 p}$ band is half-filled.

Detailed calculations show that as the unit cells evolve from compressed geometries to expanded geometries the calculated band structures change differently depending on the spin-polarization. For the MnN compound the LDA and GGA calculations carried out by taking the lattice parameter varying from $\mathrm{a}=4.10 \AA$ to $\mathrm{a}=5.00 \AA$ exhibit the same qualitative behavior. The majority-spin bands evolve from a situation in which the Fermi level is exactly on the edge of a fully occupied $\Gamma_{12}$ band to a situation in which the Fermi level cuts through the higher lying $\Gamma_{15 d}$ band, which turns to be partially occupied; at the same time the minority-spin bands evolves to a situation in which the Fermi level
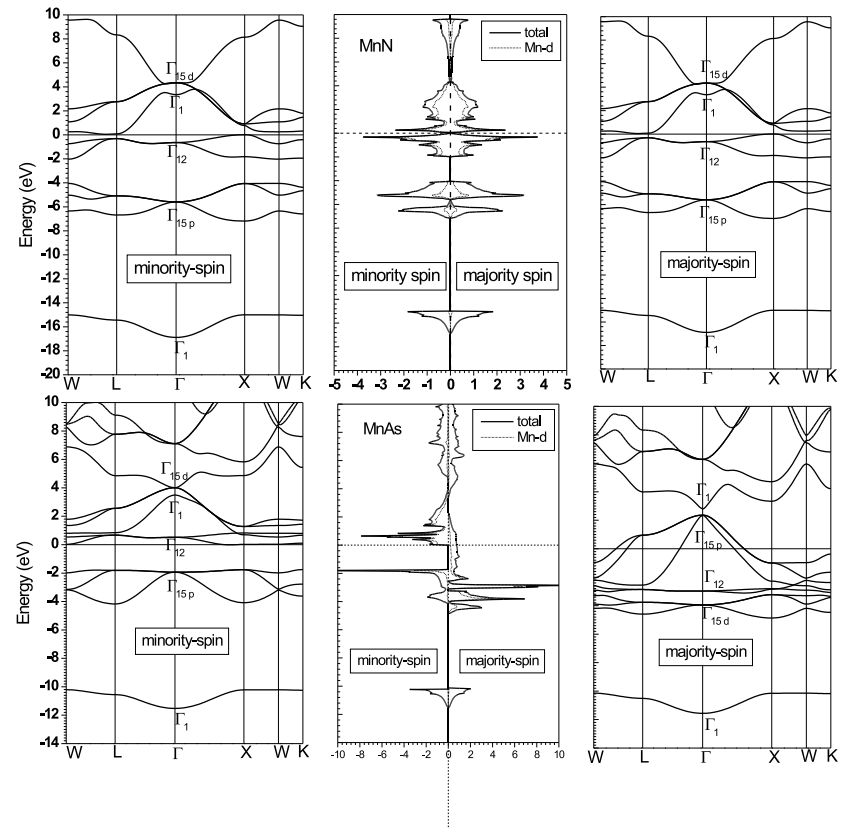

Figure 2. Electronic structures for zinc-blende $\mathrm{MnN}$ (top) and MnAs (bottom) at the GGA total energy minimum $\left(a_{o}=4.30 \AA-\right.$ $\mathrm{MnN}$ and $a_{o}=5.71 \AA-\mathrm{MnAs}$ ). The figures on the left and on the right correspond to the band structures for the minority-spin and majority-spin, respectively. The figures in the middle correspond to the density of states. The horizontal line refers to the position of the Fermi level.

just touch an empty $\Gamma_{12}$ band, being on the edge of the big gap between $\Gamma_{15 p}$ and $\Gamma_{12}$. Therefore in this range of lattice parameters the $\mathrm{MnN}$ material changes from a paramagnetic metallic phase to a ferromagnetic phase in which the majority-spin system is metallic and the minority-spin is isolating. With regard to the MnAs compound both LDA and GGA calculations also give qualitative similar results. As long as the lattice parameter is varied from $a=4.80 \AA$ to $5.90 \AA$ the majority-spin system keeps a metallic character with the Fermi level lying successively higher across the $\Gamma_{15 p}$ band; meanwhile the minority-spin system evolves from a metallic character in which the Fermi level lies in the middle of the $\Gamma_{12}$ band to a isolating character in which the Fermi level lies in the gap between the $\Gamma_{15 p}$ and $\Gamma_{12}$ bands.

\section{Conclusion}

Based on our first-principles calculations we conclude that both materials, $\mathrm{MnN}$ and $\mathrm{MnAs}$, may be half-metallic in zinc-blende stretched lattices in which $a_{o}>4.90 \AA$ for $\mathrm{MnN}$ and $a_{o}>5.71 \AA$ for MnAs, therefore showing conduction by charge carriers of one spin direction exclusively. The majority-spin band is continuous, while the minority-spin has a gap. Both materials reach a total magnetization of the order of $4 \mu_{B}$. MnN changes from paramagnetic to ferromagnetic with the increase of the lattice parameter. 


\section{Acknowledgement}

The authors are grateful to the Brazilian agencies CNPq, FAPEMIG and CAPES for financial support, and to CENAPAD-MG-CO for the computational support.

\section{References}

[1] M. Zajac, J. Gosk, M. Kaminska, A. Twardowski, T. Szyszko, and S. Podsiadlo, Appl. Phys. Lett. 79 (15), 2432 (2001).

[2] M. E. Overberg, C. R. Abernathy, S. J. Pearton, N. A. Theodoropoulou, K. T. McCarthy, and A. F. Hebard, Appl. Phys. Lett. 79 (9), 1312 (2001)

[3] M. L. Reed, N. A. El-Masry, H. H. Stadelmaier, M. K. Ritums, M. J. Reed, C. A. Parker, J. C. Roberts, and S. M. Bedair, Appl. Phys. Lett. 79 (21), 3473 (2001).

[4] N. Theodoropoulou, A. F. Hebard, M. E. Overberg, C. R. Abernathy, S. J. Pearton, S. N. G. Chu, and R. G. Wilson, Appl. Phys. Lett. 78 (22), 3475 (2001).

[5] Y. Shon, Y. H. Kwon, S. U. Yuldashev, J. H. Leem, C. S. Park, D. J. Fu, H. J. Kim, T. W. Kang, and X. J. Fan, Appl. Phys. Lett. 81 (10), 1845 (2002).

[6] A. F. Guillermet, G. Grimvall, Phys. Rev. B 40, 10582 (1989).

[7] L. Kronik, M. Jain, and J. R. Chelikowsky, Phys. Rev. B 66 , 041203 (R) (2002).

[8] E. Kulatov, H. Nakayama, H. Mariette, H. Ohta, and Yu. A. Uspenskii, Phys. Rev. B 66, 045203 (2002).

[9] M. Shirai, T. Ogwa, I. Kitagawa, and N. Suzuki, J. Magn. Magn. Mater. 177-181, 1383 (1998).
[10] S. Sanvito and N. A. Hill, Phys. Rev. B 62 , 15553 (2000).

[11] A. Continenza, S. Picozzi, W. T. Geng, and A. J. Freeman, Phys. Rev. B 64, 085204 (2001).

[12] A. Janotti, Su-Huai Wei, and Laurent Bellaiche, Appl. Phys. Lett. 82,766 (2003).

[13] K. Suzuki, T. Kaneko, H. Yoshida, Y. Obi, H. Fujimori, and H. Morita, J. Alloys Comp. 306 (1-2), 66 (2000).

[14] K. Maki, J. Kaneko, H. Hiroyoshi, and K. Kamigaki, J. Magn. And Magn. Mats. 177, 1361 (1998).

[15] K. Suzuki, Y. Yamaguchi, T. Kaneko, H. Yoshida, Y. Obi, H. Fujimori, and H. Morita, J. Phys. Soc. Jpn. 70 (4), 1084 (2001).

[16] M. Mekata, H. Yoshimura, and H. Takaki, J. Phys. Soc. Jpn. 33 (1), 62 (1972).

[17] F. Schippan, M. Kästner, L. Däweritz, and K. H. Ploog, Appl. Phys. Lett. 76 , 834 (2001).

[18] M. Mekata, J. Haruna, and H. Takaki, J. Phys. Soc. Jpn. 21, 2267 (1966).

[19] W. J. Takei, R. R. Heikas, and Shirane, Phys. Rev. 125, 1893 (1962).

[20] P. Hohenberg and W. Kohn, Phys. Rev. 136, B864 (1964); W. Kohn and L. J. Sham, ibid. 140, A 1133 (1965).

[21] P. Blaha, K. Schwarz, G. K. H. Madsen, D. Kvasnicka, and J. Luitz, Techn. Universität Wien, Austria, 2001. ISBN 39501031-1-2. 